\title{
Effect of a magnetic field on the martensitic transition of Cu-Al-Mn alloys
}

\author{
J. Marcos, A. Planes, LI. Mañosa, A. Labarta ${ }^{1}$ and B.J. Hattink ${ }^{1}$ \\ Departament d'Estructura i Constituents de la Matèria, Facultat de Física, \\ Universitat de Barcelona, Diagonal 647, 08028 Barcelona, Catalonia, Spain \\ ${ }^{1}$ Departament de Física Fonamental, Facultat de Física, Universitat de Barcelona, \\ Diagonal 647, 08028 Barcelona, Catalonia, Spain
}

\begin{abstract}
We study the effect of a magnetic field on the martensitic transition of a $\mathrm{Cu}-\mathrm{Al}-$ Mn shape memory alloy. We present resistance measurements through the martensitic transition under applied magnetic fields ranging from 0 to $50 \mathrm{kOe}$. Results show that while the onset of the transformation is only slightly affected by the magnetic field, the extension in temperature of the transition is significantly reduced upon increasing the field. This finding reflects the existence of magnetoelastic coupling at a mesoscopic scale between magnetic and structural domains.
\end{abstract}

\section{INTRODUCTION}

$\mathrm{Cu}-\mathrm{Al}-\mathrm{Mn}$ alloys with an atomic Mn content $x<25 \%$ show a bcc (or $\beta$-phase) structure which is stable at high temperatures and can be retained in a long-lived metastable state at low temperature by means of a fast enough cooling. During this cooling the system develops an $L 2_{1}$ ordered structure in a two-step process $\left(A 2 \rightarrow B 2 \rightarrow L 2_{1}\right)$ in which the Mn-atoms tend to locate in one of the four distinguishable $f c c$ sublattices [1]. Upon further cooling, a martensitic transition occurs which is at the origin of the shape-memory properties exhibited by this materials. In contrast to conventional Hume-Rothery shape-memory alloys, $\mathrm{Cu}-\mathrm{Al}-\mathrm{Mn}$ displays magnetic properties which arise from the presence of localized magnetic moments at $\mathrm{Mn}$-atoms. The stoichiometric $\mathrm{Cu}_{2} \mathrm{AlMn}$ (which is the prototypical Heusler alloy) is ferromagnetic with a relatively high Curie temperature $\mathcal{T}_{c} \simeq 630 \mathrm{~K}$ [2]. For low Mn content the system does not order ferromagnetically but rather, a spin-freezing process takes place at a relatively low temperature $[3,4]$. An interesting feature regards the fact that for $x>6 \%$, the alloys show superparamagnetic properties due to the tendency of the magnetic moments to form clusters. Actually the spin-dependent scattering of conduction electrons at these clusters could explain the magnetoresistive properties recently observed at room temperature in $\mathrm{Cu}-\mathrm{Al}-\mathrm{Mn}$ melt-spun ribbons $[5,6]$. The clustering process can be enhanced taking profit of the phase separation between $\mathrm{Cu}_{3} \mathrm{Al}$-rich and $\mathrm{Cu}_{2} \mathrm{AlMn}$-rich domains that occurs within the miscibility gap below the $L 2_{1}$ ordering line [7].

In this paper we focus on the study of the influence of magnetic degrees of freedom on the martensitic transformation. This is especially motivated by the possibility of a magnetic field control of shape-memory properties. At present it has been shown [4] that in homogeneous samples where phase separation is avoided, the magnetic contribution to the overall martensitic transition entropy change is less than $<0.1 \mathrm{~J} / \mathrm{Kmol}$ (below experimental uncertainty). This means that the strength of spin-phonon coupling is small in $\mathrm{Cu}-\mathrm{Al}-\mathrm{Mn}$. Nevertheless mesoscopic coupling between magnetic clusters and martensitic variants could occur due to a difference of the Zeeman energy in differently oriented martensitic variants. Actually, such a coupling has been demonstrated to occur in the 
ferromagnetic Ni-Mn-Ga alloy [8]. The paper is organized as follows. In section 2 we briefly describe the main characteristics of the samples used and experimental set-up. Results are presented in section 3 and in section 4 we discuss the results.

\section{EXPERIMENTAL}

Measurements have been performed on two $\mathrm{Cu}-\mathrm{Al}-\mathrm{Mn}$ polycrystals (grain size $\sim 0.1 \mathrm{~mm}$ ) prepared by melting pure elements (99.99\%). The nominal compositions are $\mathrm{Cu} ; 23.6$ at\% $\mathrm{Al} ; 6.3$ at \% $\mathrm{Mn}$ (sample $A$ ) and $\mathrm{Cu} ; 22.8$ at\% $\mathrm{Al} ; 9.0$ at $\% \mathrm{Mn}$ (sample $B$ ). On cooling from the $\beta$-phase stability region, these alloys undergo two ordering transitions at the following temperatures: $T_{c}(A 2 \rightarrow B 2)=848 K$ and $849 K$, and $T_{c}\left(B 2 \rightarrow L 2_{1}\right)=802 K$ and $795 K$ for samples $A$ and $B$ respectively. Their nominal martensitic transition temperatures $T_{M}$ are $257 \mathrm{~K}$ and $157 \mathrm{~K}$, respectively.

Rectangular shaped samples (around $12 \times 4 \times 0.5 \mathrm{~mm}^{3}$ ) have been cut from cilindrical-shaped ingots. The samples have been annealed for $900 \mathrm{~s}$ at $1073 \mathrm{~K}$, quenched into a mixture of ice and water, and aged at room temperature for several days. For electrical resistance measurements they were polished down to a thickness below $0.05 \mathrm{~mm}$. Electrical resistance measurements have been carried out in the range from 5 up to $300 K$ using a four-probe method in a cryostat with a superconducting magnet enabling the application of magnetic fields up to $5 T$.

\section{RESULTS}

Figs. $1 \mathrm{a}$ and $\mathrm{b}$ show the relative change of the electrical resistance $(R)$ as a function of the applied magnetic field $H$, for the two studied samples, at different temperatures below and above their martensitic transition. The relative change $\Delta R / R_{0}$ has been computed as:

$$
\frac{\Delta R}{R_{0}}=\frac{R(T, H)-R(T, H=0)}{R(T, H=0)}
$$

Experiments have been performed by first cooling the sample down to $10 \mathrm{~K}$ and increasing the temperature by steps and measuring resistance versus magnetic field at different temperature plateaus (the starting $T_{S}$ and finishing $T_{F}$ temperatures of the reverse transition are indicated by arrows).

For both samples $\Delta R / R_{0}$ decreases as the magnetic field increases; the magnitude of this change being higher at lower temperature. The measured values of $\Delta R / R_{0}$ are similar for both samples. However, it is apparent that sample $B$, with larger $M n$ content, behaves magnetically softer than sample $A$. These results prove that bulk polycrystalline samples display magnetoresistive properties which are perfectly comparable with those shown by melt-spun ribbon samples of similar composition [6].

An interesting feature revealed by Fig.1 regards the influence of the martensitic transition on the relative change of electrical resistance $\Delta R / R_{0}$. This quantity is increased in the martensitic phase. It is therefore interesting to analyze in more detail the influence of a magnetic field on the change of $R$ with temperature accross the transition. This can be carried out from continuous heating measurements of $R$ throught the martensitic transition at different values of the magnetic field or alternatively from constant $H$ sections of the measured $R$ vs. $H$ isotherms. We have obtained that both procedures yield to $R$ vs. $T$ curves with perfectly comparable shape. It is, however, worth noting that the obtained transition temperatures can differ slightly in both cases. The difference could have the origin in uncontrollable metastability effects. Since such undesirable effects may mask any possible change of transition temperatures with magnetic field, we have decided to study the 


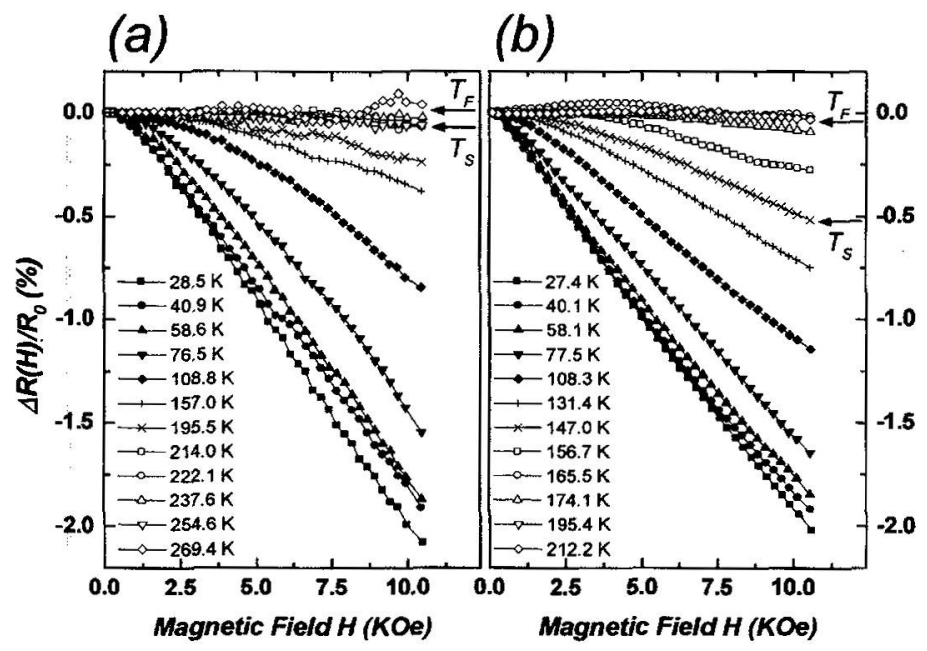

Figure 1: Relative change of resistance as a function of the applied magnetic field at different temperatures. The arrows indicate the range where the martensitic transition takes place. (a) Results for sample A. (b) Results for sample B.

influence of magnetic field from isothermal measurements which avoid temperature cycling throught the transition. Isothermal runs have been performed every $5 \mathrm{~K}$ in the range from 0 to $50 \mathrm{kOe}$.

Fig. 2 shows typical $R$ versus $T$ curves during the reverse martensitic transitions of sample $B$ at different values of the applied magnetic field. A characteristic transition temperature $T_{M}$ is given by the temperature of the minimum of the derivative $d R / d T$ (inflexion point). We have obtained that this temperature shows a small but systematic dependence on $H$ which is indicative of a small increase of transition temperature with applied magnetic field. We have estimated that this change is less than $0.005 \mathrm{~K} / \mathrm{kOe}$. More significant is the fact that the transition spreads in a narrower temperature range as the field is increased. In Fig. 3a we have plotted this range as a function of the applied magnetic field. The spread in transition temperature $\Delta T$ has been estimated as the temperature difference between the maximum and the minimum of the $R$ versus $T$ curves. Moreover, $\Delta R$ at the transition decreases with the applied field. This is shown in Fig. $3 \mathrm{~b}$. In the range of studied fields no saturation is obtained for these quantities.

\section{DISCUSSION AND CONCLUSIONS}

The influence of a magnetic field on the martensitic transition has been investigated from long ago in ferrous alloys and steels, and more recently in the Heusler $\mathrm{Ni}_{2} \mathrm{MnGa}$ ferromagnetic system. In the former case the most prominent effect is the considerable change $\Delta T_{M}(>2 \mathrm{~K} / k \mathrm{Oe})$ of the transition temperature with applied field [9]. This change is mainly accounted for by the difference $\Delta M$ in the magnetic moment of the high and low temperature phases [10]. From Clausius -Clapeyron equation it is obtained: 


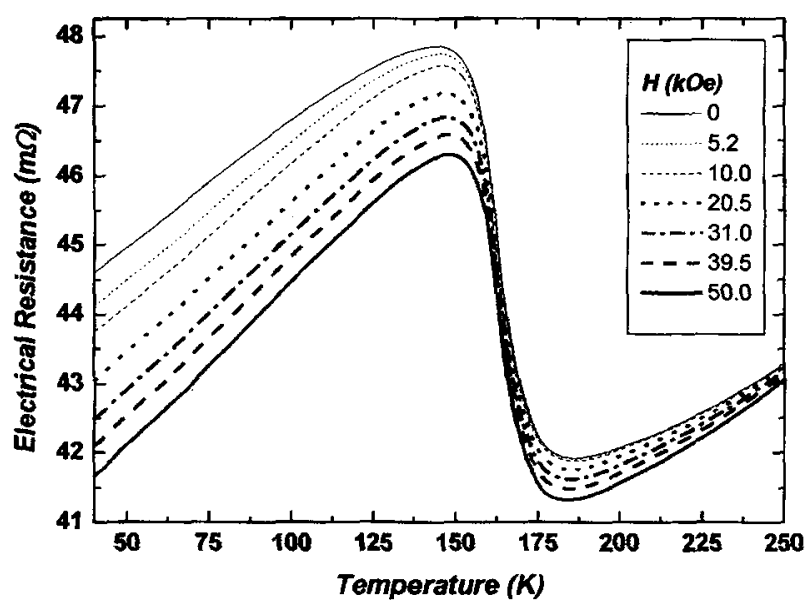

Figure 2: $R$ versus $T$ curves accross to the transition from martensite to the $\beta$-phase, at different values of the magnetic field $H$ for sample $B$.

$$
\Delta T_{M}=-\frac{\Delta M}{\Delta S} H
$$

where $\Delta S$ is the entropy change between both phases. In $\mathrm{Ni}_{2} \mathrm{MnGa}, \Delta M / \Delta S$ is rather small and $\Delta T_{M}$ depends very weakly on $H[11]$. Neverthelees the martensitic twin domain structure has been shown to be field dependent since Zeeman energy difference accross twin boundaries must be considered together with elastic energy (crystal-lattice mismatch between high and low temperature phases) and surface energy in order to determine an energetically favourable transition path [8].

In this paper we have reported preliminary results of the influence of a magnetic field on the martensitic transition of the $\mathrm{Cu}-\mathrm{Al}-\mathrm{Mn}$ shape-memory alloy. By combining the results presented here with reported values of $\Delta S$, equation 2 predicts a very small change in magnetization at the martensitic transition.

A magnetoresistive behaviour has been observed in bulk polycrystaline samples of Cu-Al-Mn. This magnetoresistance is negative, i.e the electrical resistance decreases with increasing the magnetic field. This is a typical feature of granular magnetic solids where magnetoresistance mainly originates from the spin-dependent scattering of conduction electrons at ferromagnetic clusters embbeded in a non-magnetic matrix. These results are consistent with the tendency to clustering of Mn-atoms which was first proposed by Prado and collaborators [3]. Notwithstanding, the measured magnetoresitance is small, especially in the high-temperature $\beta$-phase. The effect of the martensitic transition is to increase the resistivity of the alloy but the change of resistivity at the transition diminishes with $H$. This is mainly due to the large decrease of the resistivity of the martensitic phase with the magnetic field.

The most important effect of the magnetic field on the martensitic transition is the reduction of the temperature range in which the transition occurs. Since in polycrystalline alloys, the change of resistance with temperature provides a very good description of the corresponding evolution of 


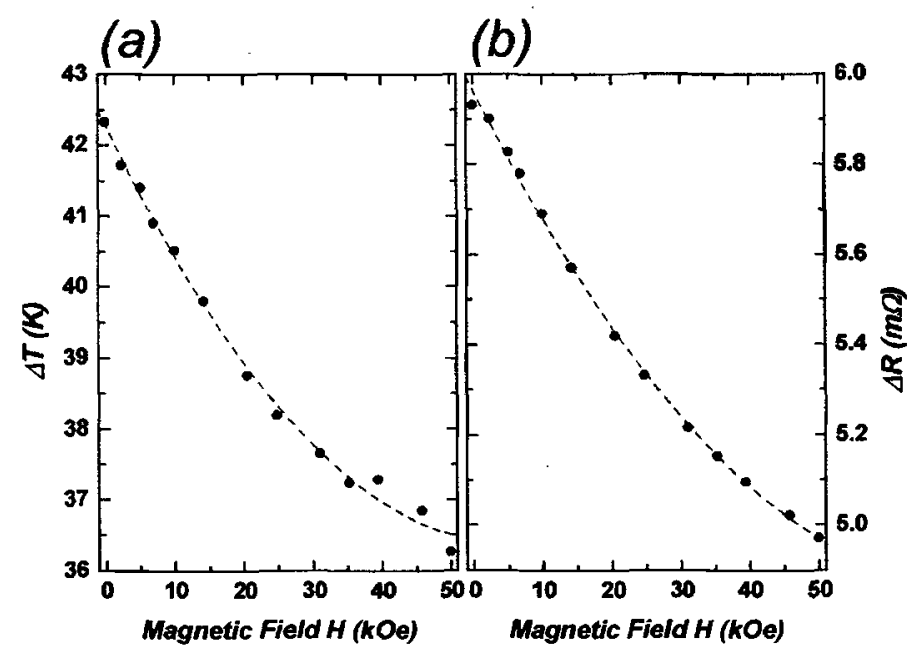

Figure 3: (a) Spread of the transition $(\Delta T)$ as a function of the applied magnetic feld. (b) Change of resistance $(\Delta R)$ at the martensitic transition, as a function of the applied magnetic field.

the transformed fraction it seems clear that the magnetic field modifies the transition path. It has been proposed that the transition temperature range $\Delta T$ is related to the stored elastic energy [12]. Therefore, a decrease in $\Delta T$ should be ascribed to a lower value of the stored elastic energy. We suggest that the physical mechanism behind this effect is the fact that in the vicinity of magnetic clusters, the magnetic field breaks the degeneracy of the low-temperature phase by favouring the nucleation of those martensitic variants with magnetization easy-axis in the direction of the field. In $\mathrm{Cu}-\mathrm{Al}-\mathrm{Mn}$, orthorrombic martensitic domains with the $c$-axis oriented along the magnetic field are expected to have maximum nucleation probability.

\section{ACKNOWLEDGEMENTS}

This work has received financial support from the CICyT (Spain), project MAT98-0315 and from the CIRIT (Catalonia), project 1998SGR48. J.M acknowledges finacial support from Direcció General de Recerca (Catalonia) under a predoctoral fellowship.

\section{References}

[1] E. Obradó, C. Frontera, LI. Mañosa, and A. Planes, Phys. Rev. B, 58, 14245 (1998).

[2] P.J. Webster and K.R.A. Ziebeck, in Magnetic Properties of Metals, edited by O. Madelung, Landolt-Börnstein, New Series, Group 3, Vol. 19, Pt. c (Springer-Verlag, Berlin, 1988), p. 75.

[3] M.O. Prado, F.C. Lovey, and L. Civale, Acta Mater., 46, 137 (1998).

[4] E. Obradó, B. Martínez, and A. Planes, Phys. Rev. B, 59, 11450 (1999). 
[5] A.S. Murthy, L. Yiping, G.C. Hadjipanayis, and K. R. Lawless, IEEE Trans. Mag., 31, 3958 (1995).

[6] S. Sugimoto, S. Kondo, H. Nakamura, D. Book, Y. Wang, T. Kagotani, R. Kainuma, K. Ishida, M. Okada, and M. Homma, J. Alloys Comp., 265, 273 (1998).

[7] M. Bouchard and G. Thomas, Acta Metall., 23, 1485 (1975).

[8] R.C. O'Handley, J. Appl. Phys., 83, 3263 (1998).

[9] T. Kakeshita, K. Shimizu, S. Funada, M. Date, Acta metall. 33, 1381 (1985).

[10] M.A. Krivoglaz and V.D. Sadovsky, Fizika Metal. Metall., 18, 4502 (1964).

[11] A. Gonzàlez-Comas, E. Obradó, Ll. Mañosa, A. Planes, V. A. Chernenko, B.J. Hattink and A. Labarta, Phys. Rev. B, 60, 7085 (1999).

[12] J. Ortín and A. Planes, Acta metall., 36 , 1873(1988). 